\title{
DIVERTICULITIS OF THE COLON
}

\author{
By Henry R. Thompson, F.R.C.S. \\ Surgeon, St. Mark's Hospital for Diseases of the Rectum and Colon, and Woodford Fubilee and Forest Hospitals
}

Diverticula of the large intestine have been recognized for the last 250 years. The first descriptions of diverticula resulted from postmortem examinations, the earliest of these being ascribed to the-French surgeon, Alexis Littré, in I700. The condition has also been described by such time-honoured morbid pathologists as Matthew Baillie, Rudolf Virchow and Jean Cruveilhier, whose writings in 1849 are generally accepted as the starting point of our better understanding of the condition. In the early part of this century clinical recognition of the condition resulted in successful resections of diseased segments of bowel by surgical giants as Lord Moynihan and W. J. Mayo of the famous American clinic. In I913 the use of contrast media in gastro-intestinal radiology gave an added impetus to our knowledge of this condition. Today, diverticulosis of the colon and its complications is frequently met with in the outpatient department and emergency service.

\section{Aetiology}

From the age incidence it must be assumed that diverticulosis is an acquired disease and this is supported by the fact that the diverticula in diverticulosis consist of mucous membrane and a serous coat only and are thus false diverticula, in contrast to congenital or true diverticula, which characteristically consist of all coats of the bowel wall.

Diverticula are herniations of the mucous membrane through the bowel wall. For this to occur two factors must be present-a weak spot in the colon wall and a raised pressure within the bowel sufficient to push the mucous membrane out. A clue to the weak spot in the colon wall is found by studying the distribution of the diverticula. They occur where the longitudinal muscle is absent and where the branches of the mesenteric vessels pass through the circular muscle coat of the colon to supply the mucous membrane. The range of intracolonic pressure is still a matter of speculation. It can, however, be noted that intestinal residue has become solid by the time it reaches the pelvic colon and it is not illogical to suppose that there is a relationship between the commonest site for diverticula and the consistency of the faeces.

It has been suggested that abnormal pressures may be produced by disorders of peristalsis. Peristalsis consists of a co-ordinated wave of contraction preceded by a wave of relaxation passing down the colon. If this rhythm becomes disordered it is possible that local intra-colonic pressures may be raised.

\section{Age and Sex Incidence}

It is unusual for diverticula to give rise to symptoms before the age of 35 . From the age of 40 onwards the incidence increases to reach its peak in the sixth decade. In the past there was said to be a preponderance of males to female suffering from the disease, but recent figures sugê gest that the incidence is about the same in both sexes.

\section{Distribution}

Although diverticula can occur throughout the colon, diverticulosis is almost invariably a problem of the pelvic or sigmoid loop of colon. A solitary diverticulum may arise in the caecum, become acutely inflamed, simulating appendicitis, or chronically inflamed, forming a hard, tender lump simulating a caecal neoplasm. Again, in the transverse colon diverticula may give rise to pericolic inflammation and abscess formation with all the signs, symptoms and radiological appearances of a carcinoma. These, however, are the exceptions.

\section{Pathological Anatomy}

Colon diverticula consist of small pouches of mucous membrane covered by peritoneum. When $N$ fully developed a diverticulum is usually 0.5 to $\mathrm{N}$ I $\mathrm{cm}$. in diameter, or about the size of the average $N$ garden pea. Very occasionally diverticula may reach a giant size. In these cases the orifice of the diverticulum is found to be obstructed by a polyp which, by valvular action, permits the entry of intestinal contents and mucus into the diverticulum but prevents their return into the lumen of the bowel. 
It is necessary now to recall that the longitudinal coat of the colon is divided into three taeniae, one corresponding to the mesenteric attachment of the colon and one on either side of the antimesenteric margin. This leaves three areas of colon wall which are deficient in longitudinal muscle - an area on the antimesenteric margin and an area on either side of the mesenteric taenia - and it is through the last two zones that diverticula occur. The reason for this is that the larger subdivisions of the mesenteric vessels pass through the circular wall of the bowel in these two areas and it is alongside these vessels that the mucous membrane protrudes. Because only the smallest vessels pass through the circular muscle of the antimesenteric surface of the bowel, diverticula are rarely seen there. At first the sac is a small, wide-mouthed structure and faecal material can enter and leave with ease. As the sac develops, however, the neck becomes drawn out and constricted and faecal material becomes trapped inside the diverticulum to form a small, hard, circumscribed pellet.

Changes are found in the muscle coats of the bowel, characterized by hypertrophy of both longitudinal and circular muscle coats, the hypertrophy varying considerably from case to case. The hypertrophy of the muscle usually extends above and below the visible diverticula and is a factor which makes intestinal anastomosis following resection for diverticulosis a difficult procedure. The result of the muscle hypertrophy is that the colon is shortened and concertina-ed, throwing the bowel wall up into a series of concentric folds and, in the more severe cases, producing a localized tumour closely resembling a carcinoma.

\section{Symptoms and Signs}

Fully developed diverticulosis can be present without giving rise to symptoms. It is a common incidental finding at laparotomy, the patient having been unaware of its presence, and it is frequently demonstrated at autopsy. The symptoms of diverticulosis are a vague, indefinite dyspepsia, consisting of discomfort, flatulence, intermittent distension and difficulty in getting the bowels open. These mild symptoms may occur intermittently over a long period of time without prompting the patient to seek medical advice. If acute complications should occur the recognition of these vague early symptoms will help in establishing a diagnosis. Patients usually seek advice for the more severe symptoms of the complications of diverticulosis, which are:

\section{Diverticulitis}

Diverticula may become inflamed early or late in the development of the disease. An inflammatory process may involve only one diverticulum or the whole segment of the affected colon. It may be acute, subacute, chronic or recurrent.

Acute diverticulitis gives rise to pain, fever, nausea, sickness, diarrhoea and the passage of blood and mucus. When situated in the pelvic colon it has been aptly described as left-sided appendicitis. The inflammation may terminate in resolution, abscess formation, fibrosis or gangrene. Although resolution may occur, once the diverticula have become inflamed recurrent attacks are to be expected.

\section{Abscesses and Fistulae}

Abscess formation in diverticulitis is usually the result of a small perforation of a diverticulum which is localized by the omentum and neighbouring abdominal viscera. It may simulate a perinephric abscess or point in the groin, presenting as a suppurative inguinal adenitis. When a diverticular abscess develops between the layers of the mesentery the abscess is difficult to locate and diagnose and cases of diverticulitis with abscess formation are not infrequently the cause of prolonged undiagnosed pyrexia. These abscesses may either resolve or rupture. Resolution of the mesenteric type of abscess may give rise to large chronic fibrotic tumours in the mesentery. Rupture of the abscess may take place into the bladder, into loops of small intestine or on to the abdominal wall, producing the corresponding fistulae. The commonest fistula is the vesico-colic, which is usually preceded by attacks of painful frequency of micturition. When the fistula between bladder and bowel is established, pneumaturia is added to the symptoms of cystitis. It should also be remembered that diverticulitis can give rise to a portal pyaemia and liver abscesses.

\section{Stricture and Intestinal Obstruction}

Diverticulitis terminating in fibrosis can produce two types of strictures-a localized area 2 to 3 in. long closely resembling a carcinoma, or a long, tubular stricture involving the whole segment of pelvic colon. Localized tumours are formed not only by fibrosis, but by contraction of the hypertrophied longitudinal muscle bunching up the involved segment of colon. Long, tubular strictures are an advanced manifestation of the disease and are characterized by a diffuse pericolic fibrosis. The symptoms produced by this kind of lesion are colic, constipation and intermittent diarrhoea, associated with abdominal distension, flatulence and borborygmi. The intestinal obstruction thus produced is rarely acute, but more of the chronic or subacute variety. When acute obstruction does occur with diverticulitis it is usually produced by a combination of obstructive and inflammatory factors. 


\section{Peritonitis}

Localized peritonitis occurs round an area of diverticulitis or a diverticular abscess and will give rise to pain and the signs of tenderness and rigidity, according to the location of the diverticulum. General peritonitis presents in two different forms: firstly, as the acute catastrophe usually associated with the perforation of a hollow viscus and the escape of its contents into the peritoneal cavity, the patient experiencing sudden, severe, generalized abdominal pain radiating up to the shoulders, followed by vomiting and prostration. The more usual form of general peritonitis in this condition, however, is that of a slow, spreading infection, resulting in a paralytic ileus and marked abdominal distension. The initial symptoms may not be severe and a diagnosis of intestinal obstruction is commonly made. The key to diagnosis in these cases is oscultation of the abdomen, where the absence of peristalsis should suggest the inflammatory nature of the ileus. A patient enjoying good health can suddenly become desperately ill or develop a paralytic ileus with disconcerting slowness due to the peritonitis of diverticulitis. It should be noted that the acute symptoms of perforation and peritonitis may occur during or shortly after defaecation and it is not difficult to appreciate how the increased intra-abdominal and intracolonic pressures during defaecation may be the final factor in rupturing a diverticulum.

\section{Haemorrhage}

Small amounts of recognizable blood may be passed per rectum in about a quarter of the patients suffering from diverticulosis and its complications. The blood may be dark and altered and mixed with mucus, being spread over the surface of the stool. It is quite unlike the bleeding from haemorrhoids, but closely simulates the bleeding from carcinoma of the colon. Very occasionally a massive haemorrhage may occur and blood transfusion may be required to save the patient's life.

\section{Diagnosis}

The diagnosis of diverticulosis of the colon and its complications depends on careful history taking and clinical examination supported by sigmoidoscopy, X-rays and clinico-pathological tests. Although statistics have been produced to show that diverticulosis occurs equally in fat and thin patients, it is the author's experience that the patient with diverticulosis presenting for treatment is invariably obese.

A common feature of a segment of colon with diverticulitis is a local deposition of fat and large appendices epiploicae and there seems to be no doubt that fatty infiltration of the colon itself may further weaken its walls and predispose to $\frac{\varrho}{3}$ diverticular formation.

\section{Sigmoidoscopy}

Sigmoidoscopy should always be performed $\stackrel{\text { के }}{\circ}$ before a radiological examination of the colon and, 듬 if possible, without any special preparation. The $\frac{\bar{c}}{\bar{c}}$ most common sigmoidoscopic finding is that at $\frac{\vec{\phi}}{\partial}$ 13 to $15 \mathrm{~cm}$. from the anal margin there is an $\varrho$ obstruction to the passage of the instrument. At is this site the mucous membrane, previously pale $\overrightarrow{0}$ salmon pink in colour, becomes red and oedematous $\overrightarrow{-}$ and attempts to pass the sigmoidoscope above this $\vec{\sigma}$ level result in pain to the patient and bleeding $\frac{\rho}{0}$ from the bowel wall. The obstruction is caused by thickening of the bowel wall itself, fixation of the colon to surrounding structures with sharp or angulation of the bowel and to the fact that the segment of colon has contracted, producing concentric folds or ridges across the lumen of the bowel. It may not be possible, even under anaes- $\frac{\rho}{7}$ thesia, for a sigmoidoscope to be passed the full $\rightarrow$ length. Diverticular openings on to the mucous $\mathbb{D}$ membrane can occasionally be seen and not infrequently the small pellet-like concretions from the diverticula may jump into the barrel of the sigmoidoscope and provide a clue to diag $\vec{c}$ nosis.

\section{$X$-ray Examination}

A barium enema may show constant spasm in a segment of the colon, irregular bowel outline (the $\stackrel{\circ}{\circ}$ ripple-border, saw-edge or pallisading effect) or $\stackrel{\mathbb{Q}}{\varrho}$ diverticula in varying numbers and at varying $\overrightarrow{\overrightarrow{0}}$ stages of development. Radiology of the colon in $\exists$ diverticulosis is assisted by giving antispasmodics, such as belladonna, banthine bromide or antrenyl $\bar{c}$ for a week prior to examination. Sometimes diverticula not demonstrated at the time of the filling and evacuation of the colon with barium may be seen in a film taken 24 hours later after the barium has been evacuated from the colon, but leaving a small residue in the diverticula.

\section{Clinico-pathological Tests}

A blood count may be of value, a leucocytosis confirming the presence of inflammatory changes $N$ or abscess formation, and the presence or absence $N$ of a secondary anaemia is of value in differentiating $N$ between diverticulosis and carcinoma. A raised $\omega$ sedimentation rate again may help to confirm diverticulitis. The occult blood test, subject to the obvious limitations, may assist in the diagnosis.

Difficulties in diagnosis occur when diverticula: give rise to inflammatory tumours in atypical situations, such as the ascending or transverse colon. 


\section{Differential Diagnosis}

Crohn's disease of the colon, amoeboma and endometrioma are rare conditions which may simulate diverticulitis. The common problem, however, is the differentiation between carcinoma of the pelvic colon and diverticulitis. It is difficult because:

(I) The conditions occur in the same age group.

(2) They give rise to the same symptoms.

(3) They are subject to the same complications.

(4) Carcinoma and diverticula may occur simultaneously in the same segment of colon.

If bleeding from the bowel is one of the symptoms and the presence of diverticula has been established, a co-existing carcinoma must be borne in mind and every effort made to exclude such a possibility.

The difficulty in making a differential diagnosis between carcinoma and a diverticular tumour is not only a clinical problem, for even at laparotomy, when the lesion is exposed, it may be impossible to distinguish between the two. This difficulty of recognition at operation arises not only when the tumour is small and mobile, but when there is a large fixed mass filling the pelvis. It is remarkable that even the most experienced surgeon is sometimes at a loss to distinguish at operation between a carcinoma and a localized lesion resulting from diverticulosis. The presence of diverticula above the lesion may indicate its nature, but, as has already been pointed out, the conditions may coexist and every so often the nature of a colon tumour remains uncertain until the pathological examination.

The wide resection of a mobile diverticular tumour in mistake for carcinoma adds little to the risk of the operation and the patient's smooth recovery. The removal of a fixed pelvic mass, however, may be a difficult operation endangering the patient's life. Where this situation exists it is far better to perform a colostomy and continue investigations after the diversion of the intestinal contents from the pathological area rather than risk performing an extensive operation for a benign condition.

\section{Treatment}

The treatment of uncomplicated diverticulosis should be medical because:

(I) Symptoms are not severe.

(2) Acute exacerbations of symptoms may be of short duration and separated by many years of freedom.

(3) It is bad surgery to operate on elderly, obese patients for a benign condition which might be controlled by medical measures.

\section{Medical Treatment}

As the aetiology of diverticulosis is still obscure there is no rational treatment. Medical measures $c$. are directed at avoiding colon irritation and $\underset{\vec{F}}{\vec{*}}$ increased intracolonic pressure and at relieving $\stackrel{0}{\sigma}$ colon spasm. Colon irritation can be lessened by a low residue diet and by excluding foods which reach the colon as hard, undigested fragments, $\frac{\rho}{\overrightarrow{0}}$ such as nuts, pips and husks. If irritation is avoided $\stackrel{\circ}{\circ}$ colon spasm is lessened.

Where colon spasm is a radiological feature, antispasmodics should be given. Regular small doses of liquid paraffin or liquid paraffin combined with milk of magnesia overcome the tendency to constipation. Most important, when the patient is fat, the doctor should insist on weight reduction and establish an exercise routine where the emphasis is on a regular daily effort made by the patient himself. A weekly Turkish bath and regular massage can assist in weight reduction and $\stackrel{\infty}{\circ}$ inspiring the patient to maintain a degree of $ᄋ$ physical fitness.

Acute diverticulitis with localized signs of peritonitis can also initially be treated medically. The patient is confined to bed, restricted to a fluid diet, phthalylsulphathiazole or neomycin sulphate is given by mouth and a close watch is kept on the patient's temperature, pulse rate and local physic signs. If in 48 to 72 hours the temperature settle and the physical signs become less, resolution cant be expected. If, however, this does not occur, the patient should be admitted to hospital in order that surgery may be at hand if required.

It is obviously bad practice to leave a condition until serious complications arise. The aim is to recognize when diverticulosis can no longer be controlled medically and when complications are likely to arise. For example, recurrent attacks of urinary symptoms suggest a diverticular abscess adjacent to the bladder wall-the precursor of a vesico-colic fistula. Such symptoms should be regarded as an indication for surgical treatment.

\section{Surgical Treatment}

The modern approach to diverticulosis and its complications has swung in favour of earlier $\frac{D}{0}$ surgical treatment. This consists of resecting the segment of affected colon. In terms of modern $N$ surgery this may seem a simple exercise. In actual i fact, resection of the colon for diverticular disease may be a most difficult operation for the following reasons:

(I) Access to the colon may be through an abdominal wall with a thick layer of subcutaneous fat.

(2) The colon is short with a correspondingly short, fatty, infiltrated mesentery and after re-

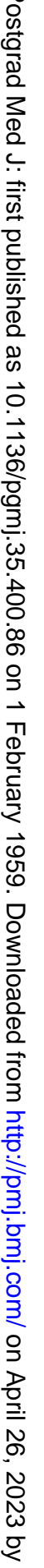


section of the diseased part there may be insufficient length of bowel or mesentery on the left side to restore continuity. In these circumstances, especially when diverticula are present in the upper descending and transverse colon, resection can be extended to include all the colon up to the hepatic flexure; the ascending colon is then rotated down and anastomosed to the upper rectum. This anastomosis proves to be very satisfactory because:

(a) The right colon can always be mobilized for anastomosis with the rectum.

(b) The resection is clear of all diverticula and the muscle coats of the bowel are not hypertrophied and difficult to suture.

(c) The lumen of the ascending colon is wide.

(d) The faecal contents of the right colon are soft or semi-fluid.

(3) The colon itself is festooned with large, fatladen appendices epiploicae, which render the preparation of a cuff of colon suitable for anastomosis extremely difficult.

(4) The longitudinal and circular muscles of the colon above and below the diverticula are thick, hypertrophied and oedematous, making inversion of the bowel edge extremely difficult and the cutting out of sutures extremely likely, however lightly they are tied.

(5) Although the maximum concentration of the diverticula may be in the pelvic colon, diverticula may be present in the descending or left half of the transverse colon in sufficient numbers to embarrass the preparation of the proximal suture line.

\section{Staging of Operation}

Although many cases of diverticular disease can be treated by a one stage resection, because of the above named difficulties it is sometimes safer to divide surgical treatment into three stages: a preliminary colostomy, followed by a variable interval during which attempts are made to reduce inflammatory changes, resection of the bowel with anastomosis and, when the patient has recovered, a closure of colostomy.

The treatment of certain complications requires special mention.

Perforation of a Diverticulum with Peritonitis. A laparotomy should be performed, the perforation identified and sutured and a right trans-rectus colostomy established. In slow-spreading peritonitis it is better to avoid the search for the perforation and only perform a colostomy.

Diverticular Abscess. There is no doubt that the establishment of a colostomy hastens the resolution of the larger abscesses resulting from diverticulosis.

Vesico-colic Fistula and the Fixed Pelvic Mass. In both these conditions a preliminary colostomy is essential to elective treatment. As regards the fixed pelvic mass of uncertain pathology, the denser and less yielding the adhesions, the more likely is the mass to prove to be diverticulitis.

After a colostomy has been established for diverticular disease it has in the past been considered sufficient to close the colostomy, after a period of six months to two years, without resecting the diseased segment of bowel. Such a step leads to a recurrence of serious complications in at least 75 per cent. of cases and is not to be recommended. Should the general condition of the patient be such that a resection of the colon is definitely contraindicated, it is better to establish a well-fashioned permanent colostomy.

\section{RUTHIN CASTLE, NORTH WALES}

A Clinic for the diagnosis and treatment of Internal Diseases (except Mental or Infectious Diseases). The Clinic is provided with a staff of doctors, technicians and nurses.

The surroundings are beautiful. The climate is mild. There is central heating throughout. The annual rainfall is 30.5 inches, that is less than the average for England.

The Fees are inclusive and vary according to the room occupied.

For particulars apply to THE SECRETARY, Ruthin Castle, North Wales. 